\title{
ABCA3 Gene Defect - Cause of Severe Respiratory Distress and Failure in Newborn
}

\author{
Neetu Yadav, Nivedita Seehra, Satish Mishra, Samar Singh \\ Department of Neonatology, Jabalpur Hospital, Jabalpur, Madhya Pradesh, India \\ Corresponding Author: Neetu Yadav
}

\begin{abstract}
Surfactant metabolism disorders are rare cause of RDS in term neonates. A near term male neonate presented with respiratory distress syndrome (required Surfactant multiple times) with family history of one still birth and one neonatal death due to RDS in previous siblings. A homozygous missense variation in exon 7 of the ABCA3 gene that results in the amino acid substitution of leucine for proline at codon 186 was detected. He died of severe respiratory failure even after multiple doses of surfactant and ventilation. Surfactant deficiency with ABCA3 gene mutation needs to be suspected in term neonate who present with respiratory distress syndrome with family history or neonatal death with respiratory distress.
\end{abstract}

Keywords: ABCA3 gene defect, Respiratory distress syndrome (RDS), neonates, neonatal death

\section{INTRODUCTION}

Respiratory distress syndrome (RDS) associated with prematurity is caused by a deficit in surfactant production and results in diffuse atelectasis, destruction of the alveolar epithelium, formation of hyaline membranes lining the small airways, inflammation, and death, if left untreated. severe RDS in infants born at $>36$ weeks of gestation, at a time when surfactant system should be functional, suggests other reasons for lung disease as differential to be considered including infectious, genetic, and /or developmental mechanisms that impair normal lung morphogenesis or surfactant production and metabolism.

\section{CASE REPORT}

A near term, male neonate born of non-consanguineous parents with family history of one still birth, one neonatal death due to respiratory distress in previous siblings. Baby required IPPR (Bag and mask ventilation) at birth, had mild respiratory distress at birth hence started on i.v. fluids and $\mathrm{O} 2$ by nasal prongs, Bubble CPAP started at 4hours of life, sepsis screen on admission was negative, in view of worsening of respiratory distress and CXR showing bilateral ground glass appearance s/o HMD, baby was ventilated and surfactant was given, patient showed significant improvement. On day-3 antibiotics were started in view of raised CRP (normal CBC and sterile culture) and increasing FIO2 and PIP requirements, 2D ECHO showed normal cardiac structures and function with marginally increased RVSP (44mmhg) suggestive of delayed physiological changes/PPHN. In view of PPHN inotropes and oral sildenafil was given. On day-4, patient had mild pulmonary ooze, repeat ECHO had $2 \mathrm{~mm}$ PDA with raised pulmonary artery systolic pressure, ibuprofen and furosemide were started. PIP and FIO2 requirement were increasing progressively and repeat $\mathrm{x}$-ray chest showed HMD like changes again hence repeat surfactant given and ventilatory settings improved drastically. Differential Diagnosis of surfactant deficiency syndrome was discussed with parents at this point, to which they were nonaccepting and refused for genetic test. 
The child again started having gradual increase of ventilatory requirements, he received surfactant again on day 9. By now the parents were convinced and agreed for genetic analysis. On day 11the child received surfactant again, the child showed improvement but it was in decreasing order with repeated instillation. Now the parents were accepting of the diagnosis and decided against surfactant, if need be arise; the ventilatory setting again started increasing and $\mathrm{x}$-ray had progressive changes of barotraumas and ABG showed hypoxia, parents decided for palliative care and requested DNR, if need arises. Baby had cardiac arrest on day 22 of life. Genetic workup came positive after two months. DNA study detected a homozygous missense variation in exon7 of ABCA3 GENE that results in amino acid substitution of leucine for proline at codon 186.

\section{DISCUSSION}

To our knowledge this is the second case of ABCA3 gene related surfactant deficiency syndrome from India. Mutation in the genes encoding the surfactant protein $B$ and $C$ (SP-B and SP-C) and the phospholipid transporter, ABCA3, are associated with respiratory distress and interstitial lung disease in the pediatric population. Expression of these proteins is regulated developmentally, increasing with gestational age, and is critical for pulmonary surfactant function at birth.

ABCA3gene mutations, which cause a type of surfactant dysfunction, lead to reduction or absence of the protein's dysfunction, lead to reduction or absence of the protein's function. Without ABCA3 protein function, the transport of surfactant phospholipid is decreased. In addition, lamellar body formation is impaired, which causes abnormal processing of SP-B and SP-C.

ABCA3 gene mutations, which cause a type of surfactant dysfunction, lead to reduction or absence of the protein's function. Without ABCA3 protein function, the transport of surfactant phospholipids is decreased. In addition, lamellar body formation is impaired, which causes abnormal processing of SP-B and SP-C.

ABCA3 Gene mutations result in abnormal surfactant composition and function. It has been suggested that mutations that eliminate ABCA3 protein function cause severe forms of surfactant dysfunctions, and mutations that leave some residual ABCA3 activity cause milder forms of the condition.

ABCA3 is a large gene located on chromosome 16 (16p13.3) and contains 30 coding exons. Mutations in the ABCA3 gene are distributed throughout its length ad consist of missense, nonsense, frame shift, and splice-site mutations as well as insertions and deletions. Lung disease caused by ABCA3 mutations is inherited as an autosomal recessive disorder. The majority of cases reported to date have been associated with surfactant deficiency, respiratory distress and failure in the neonatal period or infancy. Depending on location of the mutation in the gene, lung disease in genetic ABCA3 may be caused by a number of different mechanism, including loss of expression, decreased expression, abnormal intracellular trafficking of the protein to the lamellar body, abnormal packing of phospholipids, and/or defects in functional activity (i.e. ATP hydrolysis).

The finding of the same mutation, a substitution of valine for glutamic acid in codon 292(E292V), in multiple unrelated older patients who have ILDs suggests a genotype-phenotype correlation. A role for ABCA3 in lamellar body biogenesis is supported by electron microscopic finding in affected infants. Normal lamellar bodies are absent or markedly reduced in number; instead, small, dense bodies that have eccentrically placed very electrodense cores (giving a "fried-egg" appearance) are found in the cytoplasm of type II cells.

In our case a homozygous missense variation in exon 7 of the ABCA3 gene (chr16:g.2373580G>a;Depth:186) that results in the amino acid substitution of 
leucine for proline at codon 186 (p.pro186Leu;ENST00000301732.5) was detected. The observed variation has previously been reported as one of the compound heterozygous variants in patients affected with respiratory distress syndrome due to surfactant deficiency.

It is not possible to distinguish genetic causes of surfactant deficiency from treatable or transient causes of neonatal respiratory disease such as RDS, retained fetal lung liquid syndrome, or pneumonia purely on clinical or radiographic grounds. A Lack of risk factors for RDS or infection in a child who has diffuse parenchymal lung disease should raise suspicion for an inherited surfactant disorder, and a family history of severe neonatal lung disease should prompt evaluation. Limitations of genetic testing include cost and long turnaround times in critically ill or rapidly deteriorating infants for whom a timely diagnosis is needed for management decisions.

Acknowledgement: None

Conflict of Interest: None

\section{Source of Funding: None}

\section{REFERENCES}

1. Gower WA, Wert SE, Nogee LM. Inherited surfactant disorders. Neoreviews. 2008; 9:e458-67.

2. Shulenin S, Nogee LM, Annilo T, Wert SE, Whitsett JA, Dean M. ABCA3 gene mutations in newborns with fatal surfactant deficiency. N Engl J Med. 2004;350:1296303.

3. Hamvas A. Evaluation and management of inherited disorders of surfactant metabolism. Chin Med J. 2010;123:2943-7

4. Wert SE, Whitsett JA, Nogee LM. Genetic disorders of surfactant dysfunction. Pediatr Dev Pathol. 2009;12: 253-74.

5. Mugilan Anandarajan, Sunil Paulraj, Richard Tubman. ABCA3 Deficiency: an unusual cause of respiratory distress in the newborn. Ulster Med J 2009; 78 (1) 51-52

How to cite this article: Yadav N, Seehra N, Mishra S et.al. ABCA3 gene defect - cause of severe respiratory distress and failure in newborn. International Journal of Research and Review. 2021; 8(11): 23-25. DOI: https://doi. org/10.52403/ijrr.20211104 